\title{
Updated Cutoff Values of Basal Serum Calcitonin to Discriminate True Medullary Thyroid Cancer from Other Conditions of Hypercalcitoninemia
}

Qianhui Liu

West China Hospital of Sichuan University

Xin Nie

West China Hospital of Sichuan University

Yong He

West China Hospital of Sichuan University

Guixing Li ( $\square$ liguixing27@163.com )

West China Hospital of Sichuan University

\section{Research Article}

Keywords: Thyroid Cancer, Medullary, Diagnosis, Biomarkers, Calcitonin, Carcinoembryonic antigen

Posted Date: August 18th, 2021

DOl: https://doi.org/10.21203/rs.3.rs-690134/v1

License: (a) (i) This work is licensed under a Creative Commons Attribution 4.0 International License.

Read Full License 


\section{Abstract \\ Background}

Calcitonin (Ctn) is a tumor marker of medullary thyroid carcinoma (MTC). However, serum Ctn cutoff values for MTC diagnosis are still under discussion. This study aimed to identify the cutoff values of Ctn and explore the relationship between two tumor markers (Ctn and carcinoembryonic antigen (CEA)) and disease burden.

\section{Methods}

This was a retrospective study conducted in West China Hospital of Sichuan University. We included 61 MTC patients and 235 non-MTC patients with nonspecific elevation of Ctn $(>9.52 \mathrm{pg} / \mathrm{mL}$ for males and > $6.40 \mathrm{pg} / \mathrm{mL}$ for females) and collected patients' demographic information, essential serological indicators, cervical ultrasound and pathological reports.

\section{Results}

Unlike MTC, papillary thyroid carcinoma (40.85\%), uremia (18.73\%), chronic renal disease $(10.21 \%)$ and inflammation (8.94\%) commonly occurred with hypercalcitoninemia. The Ctn cutoff values were 38.24 $\mathrm{pg} / \mathrm{mL}$ for males and $26.00 \mathrm{pg} / \mathrm{mL}$ for females. The Ctn level was found to be positively related to the largest tumor diameter $(r=0.702)$. Serum $C$ tn levels were significantly higher in patients with lymph node metastasis than in those without $(P<0.05)$, but CEA levels did not differ $(P>0.05)$.

\section{Conclusion}

The best Ctn cutoff values for Chinese people to discriminate MTC from other hypercalcitoninemia conditions are $38.24 \mathrm{pg} / \mathrm{mL}$ for males and $26.00 \mathrm{pg} / \mathrm{mL}$ for females.

\section{Introduction}

Medullary thyroid carcinoma (MTC) originates from parafollicular C-cells of the thyroid gland that secrete polypeptide calcitonin (Ctn), accounting for $1-2 \%$ of thyroid malignancies in America[1]. MTC occurs either sporadically or hereditarily, and the latter can be detected by molecular screening for RET protooncogene mutations[2]. A hereditary pattern [multiple endocrine neoplasia type 2 (MEN 2)] is present in $20-30 \%$ of cases[3]. Although the incidence of MTC is much lower than that of other thyroid cancers, such as papillary thyroid carcinoma (PTC), it is responsible for $8-13 \%$ of the total number of deaths from thyroid malignant tumors[4]. Independent risk factors for disease-specific mortality of MTC include older age, tumor size greater than $2 \mathrm{~cm}$, and regional and metastatic disease[5]. The primary treatment of MTC is total thyroidectomy, and early surgery with complete resection of the tumor mostly determines the 
likelihood of attaining a cure for MTC[6]. If the disease spreads beyond the thyroid gland at the time of diagnosis, the cure rate will decrease, so early detection of MTC is of great significance.

For MTC patients, thyroid ultrasound examination, fine-needle nodule aspiration (FNA), serum tumor marker measurements and anatomical-pathological examination are useful diagnostic methods. Among these tests, ultrasonography lacks specificity, and FNA cytology is only able to detect approximately onehalf of MTC lesions[7]. Ctn and carcinoembryonic antigen (CEA) are two secretory products of parafollicular C-cells, and their concentrations in peripheral blood are related to MTC tumor burden, such as the largest tumor diameter, lymph node metastasis and distant metastasis. In particular, Ctn is a more specific and sensitive tumor marker for MTC than CEA. In almost all MTC patients, serum Ctn levels are increased at the time of diagnosis; the prevalence of MTC that does not secrete Ctn is only $0.83 \%[8]$. Although serum Ctn measurement is very dependent on the assay used and the normal range of the general population, it is usually recognized that if the level of basal serum Ctn is $\geq 100 \mathrm{pg} / \mathrm{mL}$, it is likely to be MTC, but if it is $<10 \mathrm{pg} / \mathrm{mL}$, the probability of developing MTC is very low[9]. When the level of serum Ctn is between 10 and $100 \mathrm{pg} / \mathrm{mL}$, Ctn stimulation tests should be performed, but a recent study showed that the Ca-Ctn stimulation test did not improve the preoperative diagnosis of MTC[10]. The determination of basal Ctn has led to a threefold increase in the diagnosis of MTC[11] and may contribute to improving the cure and survival rate of MTC[12].

Although Ctn screening is a highly sensitive tool for the diagnosis of MTC[13], the value of routine Ctn testing in patients with thyroid nodules remains unclear[14]. First, due to the low prevalence of MTC, the positive predictive value of basal Ctn testing is reduced[14]. However, many clinicians tend to prescribe serum Ctn tests for patients with thyroid nodules to avoid a missed diagnosis of MTC. The prognosis of MTC is usually poor, so early diagnosis is beneficial for its prognosis. Second, Ctn is not especially specific, and slightly moderately elevated levels of Ctn could be attributable to various confounding factors[15]. In addition to MTC, Ctn is also increased in patients who have non-MTC thyroid diseases such as autoimmune thyroiditis, follicular and papillary thyroid carcinomas and C-cell hyperplasia (CCH). Some non-thyroid diseases, such as sepsis, general inflammation, chronic renal failure and neuroendocrine tumors, will also lead to an increase in serum Ctn levels. In addition to these diseases, the level of serum $\mathrm{Ctn}$ is also influenced by age, body mass index and smoking[16]. Therefore, improving the diagnostic efficiency of serum Ctn for MTC is urgently needed. However, the serum cutoff values for any analyte depend on the assay used as well as local laboratory performance. Our study aimed to identify a cutoff value that could discriminate real MTC from other conditions of hypercalcitoninemia according to our own laboratory performance. Although the question of the clinical diagnostic value of low levels of serum Ctn has been widely discussed by several authors, the Chinese experience has never been reported. We believe that our paper is a valuable reference that should be shared with the international scientific community.

\section{Methods}

\subsection{Patients}


This was a retrospective study approved by the Ethics Committee of West China Hospital of Sichuan University and all methods were performed in accordance with the relevant guidelines and regulations. Patients who met the following inclusion criteria were included: 1) initially diagnosed with MTC and underwent thyroidectomy in our hospital between January 2011 and September 2020. 2) diagnosed with non-MTC diseases in our hospital between January 2016 and September 2020 but had elevated levels of serum Ctn (the upper limits of normal reference ranges in our laboratory were $>9.52 \mathrm{pg} / \mathrm{mL}$ for males and $>6.40 \mathrm{pg} / \mathrm{mL}$ for females). The exclusion conditions were as follows: 1 ) diagnosed with MTC but missing data on preoperative serum Ctn and CEA detection; 2) diagnosed with MTC but missing data on intraoperative or postoperative pathology; 3 ) previous surgical treatment for the thyroid gland performed at other hospitals; and 4) diagnosed with non-thyroid disease but imaging study showing suspicious occupation in the thyroid (calcification, unclear boundary, irregular shape, and increased vascularization) or suspicious lymph node in the neck (microcalcification, cystic component, peripheral vascularization, hyperechogenicity, and round shape). Finally, we included 61 MTC patients and 235 non-MTC patients and then divided them into four groups (Table 1): Group A: MTC (61); Group B1: thyroid disease except MTC (131); Group B2: kidney disease (79); and Group B3: other diseases (34). 
Table 1

Types of diseases for all 296 patients included

\begin{tabular}{|c|c|c|}
\hline Disease & Number of Patients & Proportion \\
\hline GROUP A & 61 & $100.00 \%$ \\
\hline Medullary Thyroid Carcinoma (MTC) & 58 & $95.08 \%$ \\
\hline Multiple Endocrine Neoplasia 2 (MEN2) & 3 & $4.92 \%$ \\
\hline GROUP B & 235 & $100.00 \%$ \\
\hline Group B1 (Thyroid Disease except MTC) & 131 & $55.74 \%$ \\
\hline Papillary Thyroid Carcinoma (PTC) & 96 & $40.85 \%$ \\
\hline PTC with Hashimoto's thyroiditis(HT) & 15 & $6.38 \%$ \\
\hline Hashimoto's thyroiditis(HT) & 2 & $0.85 \%$ \\
\hline Hyperparathyroidism & 7 & $2.98 \%$ \\
\hline Goiter/Adenoma & 11 & $4.68 \%$ \\
\hline Group B2 (Kidney Disease) & 70 & $29.79 \%$ \\
\hline Chronic Renal Disease (CRD) & 15 & $6.38 \%$ \\
\hline CRD complicating with Secondary Hyperparathyroidism & 5 & $2.13 \%$ \\
\hline CRD complicating with Triad Hyperparathyroidism & 4 & $1.70 \%$ \\
\hline Uremia & 10 & $4.26 \%$ \\
\hline Uremia complicating with Secondary Hyperparathyroidism & 16 & $6.81 \%$ \\
\hline Uremia complicating with Triad Hyperparathyroidism & 18 & $7.66 \%$ \\
\hline Diabetic Nephropathy & 2 & $0.85 \%$ \\
\hline Group B3 (Other Diseases) & 34 & $14.47 \%$ \\
\hline Inflammation & 21 & $8.94 \%$ \\
\hline Lung/Mediastinal/Bronchial Malignancies & 4 & $1.70 \%$ \\
\hline Intestinal Malignancies & 3 & $1.28 \%$ \\
\hline Prostate Cancer & 1 & $0.43 \%$ \\
\hline Osteonecrosis of the Femeral Head & 1 & $0.43 \%$ \\
\hline Atrial Fibrillation & 1 & $0.43 \%$ \\
\hline Pituitary Adenoma & 1 & $0.43 \%$ \\
\hline Pheochromocytoma & 2 & $0.85 \%$ \\
\hline
\end{tabular}




\subsection{Methods}

Peripheral blood samples were obtained from all patients in the early morning after fasting. For patients who underwent thyroidectomy, blood samples were collected 1 or 2 days before the operation. We collected information on patient age and sex, preoperative ultrasonographic and fine-needle aspiration (FNA) cytology reports, preoperative serological indexes (including Ctn, CEA, thyroid stimulating hormone (TSH), free triiodothyronine (FT3), free thyroxin (FT4), thyroglobulin antibody ( $\mathrm{TgAb}$ ), thyroid peroxidase antibody (TPOAb), parathyroid hormone (PTH), serum calcium (Ca), estimated glomerular filtration rate (eGFR), and serum creatinine), surgical details, and intraoperative or postoperative pathological results.

\subsection{Surgical treatment}

Patients with indications for thyroid surgery underwent operations performed by three surgeons with more than 15 years of thyroid surgery experience. Total thyroidectomy with bilateral central neck dissection was performed in all 61 MTC patients and 91 PTC patients. Total thyroidectomy was performed in 5 triad hyperparathyroidism and 5 goiter/adenoma patients, respectively. Partial thyroidectomy with unilateral central neck dissection was performed on 20 PTC patients. Additional lateral neck dissections were performed according to preoperative imaging studies, the levels of serological indicators, and intraoperative morphological appearance. Partial thyroidectomy was performed in 6 goiter/adenoma and 2 triad hyperparathyroidism patients, respectively. Parathyroid adenoma resection was performed in 7 primary hyperparathyroidism, 5 secondary hyperparathyroidism, and 8 triad hyperparathyroidism patients.

\subsection{Laboratory analysis}

Serum Ctn and CEA measurements were performed using the electroluminescent method. Detection kits and instruments (cobas e 601 and cobas e 801 modules) for Ctn and CEA were purchased from Roche Diagnostics GmbH. Scopes of detection for Ctn and CEA were $0.5-2000 \mathrm{pg} / \mathrm{mL}$ and $0.3-1000 \mathrm{ng} / \mathrm{mL}$, respectively. To meet the needs of some clinicians, we used dilutions of 100x for Ctn and 50x for CEA, and the upper limits of reporting for Ctn and CEA were $200000 \mathrm{pg} / \mathrm{mL}$ and $50000 \mathrm{ng} / \mathrm{mL}$, respectively.

\subsection{Neck ultrasonography}

Neck ultrasound examinations were performed using GE Logiq 9 and Philips iU22 color Doppler ultrasound diagnostic instruments, and probe frequency was $5 \sim 12 \mathrm{MHz}$. In 192 patients with thyroid diseases, except 157 patients that underwent total thyroidectomies, 33 patients had subtotal thyroidectomies (20 patients with PTC, 6 patients with goiter/adenoma, and 7 patients with hyperparathyroidism) and 2 Hashimoto's thyroiditis (HT) patients didn't had surgeries. Of these 35 patients who didn't have total thyroidectomy, thyroid nodules in residual thyroid were detected in 5 , and these nodules had clear boundaries, regular shapes, homogeneous echoes and no abnormal blood flow signal, which were reported as nodular goiters, grade TI-RADS 1. Of the non-thyroid disease patients, thyroid nodules were detected in 27 , and these nodules had clear boundaries, regular shapes, 
homogeneous echoes and no abnormal blood flow signal, which were reported as nodular goiters. Until the end of the study, these non-thyroid disease patients had no definite evidence of thyroid malignancy.

\subsection{Statistical analysis}

All continuous variables were tested for a normal distribution by the Kolmogorov-Smirnov (K-S) normality test. Then, normally distributed variables were expressed as the mean \pm standard deviation (M \pm SD), and skewed variables were expressed as the median (interquartile range, IQR). The differences between the two groups were examined by unpaired two-tailed Student's t-tests and Mann-Whitney Utests for normally and nonnormally distributed parameters, respectively. Categorical variables were compared by the chi-square test. Receiver operating characteristic (ROC) curves were used to evaluate the diagnostic effect of serum Ctn. Correlations between MTC patients' preoperative Ctn and CEA serum levels and intraoperative/postoperative pathological indicators (the largest tumor diameters and lymph node metastases.) were determined by calculating the Spearman correlation coefficient. P values of < 0.05 were considered to be statistically significant. All statistical analyses were performed using SPSS 25.0.

\section{Results}

\subsection{Non-MTC disease distribution}

The distribution of non-MTC diseases is shown in Fig. 1. Papillary thyroid carcinoma (PTC) accounted for $40.85 \%$ of all non-MTC diseases. PTC combined with Hashimoto's thyroiditis (HT) and HT only accounted for $7.23 \%$ of the total. In addition to the PTC and HT patients, 7 hyperparathyroidism patients $(2.98 \%)$ and 11 thyroid goiter/adenoma patients $(4.68 \%)$ also had elevated serum Ctn to varying degrees. Among these 18 patients, 17 had only mild elevation of serum Ctn, with the highest level being $14.9 \mathrm{pg} / \mathrm{mL}$. However, one 59-year-old female patient's preoperative serum Ctn level was $59.9 \mathrm{pg} / \mathrm{mL}$, and she was diagnosed with thyroid adenoma. Then, we reviewed her medical history and found that her chief complaint was neck enlargement for nine years. Her preoperative serum TSH, FT3, FT4, TgAb, TPOAb, and CEA levels were all at normal levels, but the levels of thyroid globulin $(\mathrm{Tg})$ and Ctn were high, 119.5 $\mu \mathrm{g} / \mathrm{L}$ (reference scope: 3.5-77 $\mu \mathrm{g} / \mathrm{L}$ ) and $59.9 \mathrm{pg} / \mathrm{mL}$, respectively. After total thyroidectomy, her $\mathrm{Tg}$ and Ctn decreased to very low levels $-0.11 \mu \mathrm{g} / \mathrm{L}$ and $1.6 \mathrm{pg} / \mathrm{mL}$, respectively. The postoperative pathological results did not support malignant tumors. It should also be noted that the kidney function of this female patient was normal, but she was positive for hepatitis B antigen. Kidney diseases including uremia and chronic renal disease accounted for $29.79 \%$ of non-MTC diseases with hypercalcitoninemia, and most of them were complicated with secondary/triad hyperparathyroidism. Inflammation accounted for $8.94 \%$, and various neuroendocrine tumors also led to elevation of serum Ctn.

\subsection{Basic patient information and serum Ctn comparison}

In total, 296 patients meeting the inclusion and exclusion criteria were included in our study. According to the types of diseases, these 296 patients were divided into 4 groups: Group A: MTC, Group B1: thyroid 
disease except MTC, Group B2: kidney disease, and Group B3: other diseases. Information on the 61 MTC patients is displayed in Table 2. The basic information of the four groups and their serum Ctn levels are displayed in Table 3 and Fig. 2. The staging criteria for lymph node metastasis were according to the eighth edition of the American Joint Committee on Cancer (AJCC) TNM staging system.

Table 2

Basic and clinical information of 61 MTC patients

\begin{tabular}{|ll|}
\hline Number of MTC patients & 61 \\
\hline Sex (male\%) & $23 / 38(37.70 \%)$ \\
\hline Age (years) & $46.37 \pm 11.75$ \\
\hline Number of MEN2 & $3(4.92 \%)$ \\
\hline Serological indicators & \\
\hline Level of preoperative serum Ctn $(\mathrm{pg} / \mathrm{mL})$ & $856.50(1474.15)$ \\
\hline Level of preoperative serum CEA $(\mathrm{ng} / \mathrm{mL})$ & $28.07(89.13)$ \\
\hline TgAb positive $(\geq 115 \mathrm{IU} / \mathrm{mL})$ & $4(6.56 \%)$ \\
\hline TPOAb positive $(\geq 34 \mathrm{IU} / \mathrm{mL})$ & $3(4.92 \%)$ \\
\hline Total thyroidectomy & $61(100 \%)$ \\
\hline Number of lesions & \\
\hline 1 & $53(86.86 \%)$ \\
\hline 2 & $6(9.84 \%)$ \\
\hline$\geq 3$ & $2(3.28 \%)$ \\
\hline The largest tumor diameter $(\mathrm{cm})$ & $1.50(2.10)$ \\
\hline Lymph node metastasis & $2(3.28 \%)$ \\
\hline N0 & $22(36.07 \%)$ \\
\hline N1a & $19(31.15 \%)$ \\
\hline N1b & \\
\hline Distant metastasis & \\
\hline
\end{tabular}


Table 3

The basic information and the levels of serum Ctn of the 4 groups

\begin{tabular}{|c|c|c|c|c|c|}
\hline Group & A & B1 & B2 & B3 & $P$ \\
\hline Age(years) & $46.37 \pm 11.75$ & $\begin{array}{l}40.19 \pm \\
11.64\end{array}$ & $51.40 \pm 14.95$ & $53.53 \pm 18.30$ & $>0.05$ \\
\hline $\begin{array}{l}\text { Gender(male/female) } \\
\text { (male\%) }\end{array}$ & $\begin{array}{l}23 / 38 \\
(37.70 \%)\end{array}$ & $\begin{array}{l}63 / 68 \\
(48.09 \%)\end{array}$ & $\begin{array}{l}53 / 17 \\
(75.71 \%)\end{array}$ & $\begin{array}{l}29 / 5 \\
(85.29 \%)\end{array}$ & $<0.05$ \\
\hline $\begin{array}{l}\text { Serum Ctn }{ }^{a} \\
(\mathrm{pg} / \mathrm{mL})\end{array}$ & $856.50(1474.15)$ & $10.09(4.80)$ & 16.34(13.35) & $13.50(7.45)$ & $<0.05$ \\
\hline
\end{tabular}

\subsection{Clinical value of serum Ctn in predicting MTC in patients with high serum Ctn levels}

To evaluate the predictive value of serum Ctn level for MTC, ROC curves were generated for male and female patients (Fig. 3). The AUC of serum Ctn for predicting MTC in patients with high levels of serum Ctn was 0.964 (95\% Cl: 0.898-1.000) in males and 0.998 (95\% Cl: 0.994-1.000) in females. When the Youden index had a maximum value, the sensitivity and specificity of serum Ctn for predicting MTC in males were $95.70 \%$ and $95.90 \%$, and the cutoff value was $38.24 \mathrm{pg} / \mathrm{mL}$; the sensitivity and specificity of serum Ctn for predicting MTC in females were $100.00 \%$ and $96.70 \%$, and the cutoff value was 26.00 $\mathrm{pg} / \mathrm{mL}$. For males, the sensitivity, specificity, positive predictive value (PPV), and negative predictive value (NPV) of the cutoff value $(38.24 \mathrm{pg} / \mathrm{mL}$ ) were $95.65 \%, 96.55 \%, 81.48 \%$ and $99.29 \%$, respectively. For females, the sensitivity, specificity, PPV, and NPV of the cutoff value $(26.00 \mathrm{pg} / \mathrm{mL})$ were $100.00 \%$, $97.78 \%, 95.00 \%$ and $100.00 \%$, respectively.

\subsection{The relationship between serum Ctn and CEA levels and MTC stage}

We subdivided the MTC patients in Group A into 4 groups depending on the largest tumor diameters in the pathological reports: Group a $(0 \mathrm{~cm}<$ the largest tumor diameter $\leq 1 \mathrm{~cm})$, Group b $(1 \mathrm{~cm}<$ the largest tumor diameter $\leq 2 \mathrm{~cm})$, Group $\mathrm{c}(2 \mathrm{~cm}<$ the largest tumor diameter $\leq 4 \mathrm{~cm})$, and Group $\mathrm{d}$ (the largest tumor diameter $>4 \mathrm{~cm}$ ). The serum Ctn level was significantly higher in Group b than in Group a and significantly higher in Group $\mathrm{c}$ than in Group b (Table 4, Fig. 4a). There was no significant difference in the level of serum CEA between Group a and Group b or between Group c and Group d. However, the level of serum CEA was significantly higher in Group c than in Group b (Table 4, Fig. 4b). Spearman correlations were performed to evaluate the relationships between Ctn and the largest tumor diameters, CEA and the largest tumor diameters (Fig. 4c, 4d), and the Spearman correlation coefficients were 0.702 and 0.377 , respectively. 
Additionally, we divided MTC patients into 3 groups according to lymph node staging. The serum Ctn level was significantly higher in Group N1a than in Group N0, but there was no significant difference in the serum CEA level between the two groups. However, the level of serum CEA was significantly higher in Group N1b than in Group N1a (Table 5, Fig. 5).

Table 4

The levels of serum biomarkers among groups with different tumor sizes

\begin{tabular}{|c|c|c|c|c|c|}
\hline $\begin{array}{l}\text { The largest } \\
\text { tumor } \\
\text { diameter(cm) }\end{array}$ & $0 \sim 1$ & $1 \sim 2$ & $2 \sim 4$ & $\square 4$ & $\begin{array}{l}P \\
\text { value }\end{array}$ \\
\hline $\begin{array}{l}\text { Preoperative } \\
\text { serum Ctn } \\
(\mathrm{pg} / \mathrm{mL})\end{array}$ & 285.35(548.12) & $652.7(658.5)$ & 1279(949.00) & $1525.50(2312.88)$ & $\triangle 0.001$ \\
\hline $\begin{array}{l}\text { Preoperative } \\
\text { serum CEA } \\
(\mathrm{ng} / \mathrm{mL})\end{array}$ & 12.45(19.31) & 18.83(31.49) & 67.16(137.86) & $214.55(270.00)$ & $\llbracket 0.001$ \\
\hline
\end{tabular}

Table 5

The levels of serum biomarkers among groups with different lymph node stages

\begin{tabular}{|lllll|}
\hline Lymph node staging & N0 & N1a & N1b & $\begin{array}{l}\text { P } \\
\text { value }\end{array}$ \\
\hline $\begin{array}{l}\text { Preoperative serum Ctn } \\
(\mathrm{pg} / \mathrm{mL})\end{array}$ & $293.40(960.28)$ & $1051.00(1491.7)$ & $1183.00(1183.43)$ & $<$ \\
\hline $\begin{array}{l}\text { Preoperative serum CEA } \\
(\mathrm{ng} / \mathrm{mL})\end{array}$ & $18.83(20.46)$ & $33.88(106.54)$ & $53.18(250.47)$ & $>$ \\
\hline
\end{tabular}

\section{Discussion}

Serum Ctn has always been regarded as a sensitive tumor marker, which suggests that the thyroid nodule could be MTC. There is much evidence that the measurement of serum Ctn concentrations in patients with thyroid nodules can lead to an earlier diagnosis of MTC[17]. In our research, we chose all the hypercalcitoninemia patients as the research subject, and aimed to find the best cutoff value of basal serum Ctn to discriminate MTC from other conditions of hypercalcitoninemia. It's because that from the data of all the MTC patients diagnosed and treated in West China Hospital of Sichuan University, the lowest level of serum Ctn was $10.50 \mathrm{pg} / \mathrm{mL}$, which is higher than the upper limit for our laboratory testing $(>9.52 \mathrm{pg} / \mathrm{mL}$ for males and $>6.40 \mathrm{pg} / \mathrm{mL}$ for females), so the negative predictive value (NPV) of the serum Ctn levels lower than our laboratory's lower limit of detection was $100 \%$.

According to our statistics, in addition to MTC, many other common clinical diseases can cause the elevation of serum Ctn, such as non-MTC thyroid diseases, kidney diseases, inflammation, lung and intestinal malignancies, which reduces the diagnostic specificity of serum Ctn for MTC. This result is consistent with previous literature[18], but the proportion of these diseases with hypercalcitoninemia has 
rarely been reported. Most patients who have non-MTC thyroid diseases and elevated Ctn levels (male > $9.52 \mathrm{pg} / \mathrm{mL}$, female $>6.40 \mathrm{pg} / \mathrm{mL}$ ) are diagnosed with papillary thyroid carcinoma (PTC), accounting for $40.85 \%$ of all non-MTC diseases. The reasons may be as follows: 1) PTC tumors may release some substances that possess a paracrine stimulatory action on C-cells to ultimately raise serum Ctn levels[19]; 2) PTC accounts for the vast majority of thyroid malignancies, and clinicians tend to prescribe several serum Ctn tests for patients with thyroid nodules to aid in determining the nature of the nodules. In addition to PTC, PTC combined with Hashimoto's thyroiditis (HT) or only HT (accounting for $7.23 \%$ of the total) could also cause elevated levels of serum Ctn, and this phenomenon may be a consequence of progressive C-cell destruction following the nonspecific follicular and parafollicular cell damage caused by lymphocytic infiltration of the thyroid gland[20]. In addition to thyroid diseases, kidney diseases including uremia, chronic renal disease, triad or secondary hyperparathyroidism, and diabetic nephropathy can also lead to nonspecific elevation of serum Ctn. It has been reported that $30-46 \%$ of chronic kidney disease (CKD) patients have elevated serum Ctn levels[21]. The mechanisms by which serum Ctn is elevated in these CKD patients are not yet fully understood. It is assumed that CKD patients' $\mathrm{C}$-cells are triggered by disturbances in mineral metabolism due to renal failure; thus, C-cells reactively secrete Ctn into the peripheral blood. Patients with neither thyroid disease nor kidney disease can also have elevated levels of serum Ctn due to inflammation, lung/mediastina/bronchial malignancies, intestinal malignancies and prostate cancers. In our study, inflammation accounted for the majority of this group (Group B3, other diseases), which may be due to the release of procalcitonin in the circulation by tissues that do not normally express the Ctn gene during sepsis or other inflammatory conditions[22].

Therefore, physicians must keep in mind that elevation of serum Ctn does not necessarily indicate MTC because many physiological, pharmacological, and pathological factors can influence the level of serum Ctn. An accurate medical history, physical examination and more in-depth investigation can be useful in identifying other reasons for the hyperproduction of serum Ctn[18]. However, we found that non-MTC diseases and conditions usually cause relatively lower Ctn elevation than MTC, and we thought a serum Ctn cutoff value higher than the reference range may better help to discriminate real MTC from other conditions of hypercalcitoninemia. For this reason, we included all MTC patients in West China Hospital of Sichuan University from the past decade as the test group and all non-MTC patients whose serum Ctn level was $>9.52 \mathrm{pg} / \mathrm{mL}$ (males) and $>6.40 \mathrm{pg} / \mathrm{mL}$ (females) in West China Hospital of Sichuan University from the past five years as the control group. It is recognized that serum Ctn levels are usually higher in males than in females because men have twice as many C-cells as women. Therefore, we drew different ROC curves for males and females to find sex-specific Ctn cutoffs. Finally, we determined $38.24 \mathrm{pg} / \mathrm{mL}$ (AUC: $0.964,95 \%$ Cl: $0.898-1.000$ ) and $26.00 \mathrm{pg} / \mathrm{mL}$ (AUC: $0.998,95 \% \mathrm{Cl}: 0.994-1.000$ )) to be the best cutoff values to diagnose MTC for males and females, respectively.

Among males, there was only one MTC patient whose serum Ctn level was less than $38.24 \mathrm{pg} / \mathrm{mL}$ (Ctn: $10.5 \mathrm{pg} / \mathrm{mL}$ ), and according to his postoperative pathological results, the largest tumor diameter was 1.5 $\mathrm{cm}$, without lymph node metastasis, which suggested that this patient had early-stage disease. There were five non-MTC male patients whose serum Ctn levels were higher than $38.24 \mathrm{pg} / \mathrm{mL}$. Two of them had uremia complicated by triad hyperparathyroidism (Ctn: $45.91 \mathrm{pg} / \mathrm{mL}$ and $47.8 \mathrm{pg} / \mathrm{mL}$ ). The other 
three were diagnosed with CKD4 (Ctn: $55.2 \mathrm{pg} / \mathrm{mL}$ ), severe pneumonia (Ctn: $41.9 \mathrm{pg} / \mathrm{mL}$ ), and pheochromocytoma (Ctn: $51.1 \mathrm{pg} / \mathrm{mL}$ ). The pheochromocytoma patient had no imaging evidence of thyroid nodules, thus excluding the diagnosis of MEN2. Among females, there were no MTC patients whose serum Ctn level was less than $26.00 \mathrm{pg} / \mathrm{mL}$. However, there were 2 female non-MTC patients whose serum Ctn levels were higher than $26.00 \mathrm{pg} / \mathrm{mL}$. One of them was diagnosed with CKD4 accompanied by secondary hyperparathyroidism (Ctn: $44.8 \mathrm{pg} / \mathrm{mL}$ ), and the other had a bronchial/pulmonary malignancy (Ctn: $77.73 \mathrm{pg} / \mathrm{mL}$ ). It is known that neuroendocrine tumors such as tumors predominantly of bronchial or pancreatic origin can result in inappropriate calcitonin release, which can reach $>100 \mathrm{pg} / \mathrm{mL}[23]$. Our thresholds of serum Ctn to diagnose MTC (male: $\geq 38.24 \mathrm{pg} / \mathrm{mL}$, female $\geq 26.00 \mathrm{pg} / \mathrm{mL}$ ) are comparable to the Ctn cutoff values reported in other studies (male: $\geq 46$ $\mathrm{pg} / \mathrm{mL}, 68 \geq \mathrm{pg} / \mathrm{mL},>34 \mathrm{pg} / \mathrm{mL},>43 \mathrm{pg} / \mathrm{mL}$; female: $\geq 35 \mathrm{pg} / \mathrm{mL}, \geq 18.7 \mathrm{pg} / \mathrm{mL}, \geq 26 \mathrm{pg} / \mathrm{mL},>30$ $\mathrm{pg} / \mathrm{mL},>23 \mathrm{pg} / \mathrm{mL})[24-27,10]$. The cutoff value for males in our study was slightly lower than that in the other studies mentioned above, which may be due to the following reasons: 1) We chose all patients with non-MTC diseases for the control group, whereas the other studies chose patients with $\mathrm{C}$ cell hyperplasia $(\mathrm{CCH})$ or thyroid nodule diseases for the control group, and these diseases may show higher levels of serum Ctn. 2) Different races were studied: our patients were all of Han nationality in China, while the research subjects of the other studies were mainly Caucasian or Indian. 3) Different laboratories and the use of different instruments and reagents may cause slight differences in the test results for Ctn. The method used for serum Ctn testing in our laboratory is electrochemiluminescence immunoassay (ECLIA), and the instruments and reagents are purchased from Roche Diagnostics Corp.

The preoperative serum Ctn level not only has diagnostic value but also is closely associated with primary tumor size and the number of lymph node metastases (LNMs)[28]. In our study, we found that the level of preoperative serum Ctn positively correlated with the largest tumor diameter $(r=0.702, P<$ 0.0001), which was consistent with the conclusion of Park $\mathrm{H}$ et al[28]. However, the relationship between the largest MTC tumor diameter and serum CEA level was not as strong as the largest tumor diameter and $C \operatorname{tn}(r=0.377, p=0.0025)$. We also found that the serum Ctn level was more valuable than the serum CEA level when the largest tumor diameter was less than $2 \mathrm{~cm}$, indicating that Ctn had more diagnostic value in the early stage of MTC. In a study[29] that compared the diagnostic value of four selected markers, Ctn, procalcitonin, chromogranin A, and CEA, the diagnostic value of CEA was much lower than that of the other markers and could be within the normal range even in patients with advanced metastatic MTC. Additionally, the serum CEA level was not significantly different between MTC patients with lymph node metastasis and those without lymph node metastasis $(P>0.05)$ in our study. However, the serum Ctn level in MTC patients with lymph node metastasis was significantly higher than that in patients without lymph node metastasis $(P<0.05)$. These results can be explained by the fact that $C$ tn is specifically secreted by thyroid parafollicular C-cells, but CEA is a broad-spectrum tumor marker that is not specific to MTC. For MTC patients, the diagnostic value of CEA alone is low, so CEA should serve as an auxiliary indicator. However, it has been suggested that the determination of serum CEA levels is useful for evaluating disease progression in patients with clinically evident MTC and for monitoring patients following thyroidectomy[1]. Recently, Chen Li et al reported one case in which the CEA level 
continued to increase and the Ctn value was normal after total thyroidectomy and central lymph node dissection in an MTC patient[30]. Thus, we think that CEA is more valuable for the prognosis of MTC than for its diagnosis. In the study of Meijer JAA et al[31], the CEA doubling time had a higher predictive value than Ctn for MTC recurrence and death. Therefore, more follow-up studies are needed to confirm the value of CEA in the prognosis of MTC patients.

Although clinicians in our nation tend to assess serum Ctn in patients suspected of having MTC, the cutoff value of serum Ctn for discriminating real MTC from other conditions of hypercalcitoninemia has not been reported by Chinese scholars. Serum Ctn levels can be influenced by the testing laboratory and ethnicity, so our study provides valuable information for clinical decision-making. West China Hospital of Sichuan University is a large medical center that receives patients from all over the country, and considering the very low incidence of MTC, this is a relatively large sample size that has a certain representativeness among Chinese people. We hope that our results can be a reference for clinicians in China and East Asia and for other laboratories worldwide that use ECLIA for serum Ctn testing and the same instruments and reagents offered by Roche Diagnostics Corp. In addition to calculating the cutoff value for Ctn, we also describe other diseases that can cause elevation of Ctn and have compared the diagnostic effectiveness of Ctn and CEA in MTC. Finally, we discuss the relationship between the level of serum Ctn and the tumor size and lymph node metastasis of MTC.

There are some limitations to our study. Because this was a retrospective study of MTC patients in our hospital over the past decade, the postoperative pathological reports of some patients were not available because of the prolonged study period, so we had to use their intraoperative pathological records and thyroid ultrasound reports to judge the largest tumor diameters and lymph node metastasis instead of the postoperative reports, which may be discrepant with the true pathological results. In most cases, our laboratory reported $>2000 \mathrm{pg} / \mathrm{mL}$ rather than specific values when the level of serum Ctn exceeded 2000 $\mathrm{pg} / \mathrm{mL}$, and dilution was performed only at the specific request of the clinician. For statistical convenience, we defined all values over 2000 as 2000 . This may influence the relevance analysis of Ctn and the largest tumor size and the degree of lymph node metastasis when the level of Ctn $>2000 \mathrm{pg} / \mathrm{mL}$ but had no influence on the determination of the cutoff value of Ctn when the value was at a lower level. Finally, because of the very low incidence of MTC, we were unable to find a suitable validation group in our hospital to verify the cutoff values, but subsequently, we have begun to collaborate with other hospitals to collect more MTC patients and verify the reliability of the cutoffs.

\section{Conclusion}

In our study, the best Ctn cutoff values for Chinese people to discriminate MTC from other hypercalcitoninemia conditions are $38.24 \mathrm{pg} / \mathrm{mL}$ for males and $26.00 \mathrm{pg} / \mathrm{mL}$ for females. Papillary thyroid carcinoma, kidney disease complicating with secondary/triad hyperparathyroidism and inflammation are common non-MTC diseases leading to hypercalcitoninemia. In MTC patients, serum Ctn is a better biomarker than CEA to reflect the tumor burden. 


\section{Abbreviations}

Ctn, calcitonin;

MTC, medullary thyroid cancer;

CEA, carcinoembryonic antigen;

MEN 2, multiple endocrine neoplasia type 2;

PTC, papillary thyroid carcinoma;

FNA, fine-needle nodule aspiration;

$\mathrm{CCH}$, C-cell hyperplasia;

HT, Hashimoto's thyroiditis;

Tg, thyroid globulin;

CKD, chronic kidney disease

\section{Declarations}

\section{Acknowledgements}

The authors would like to acknowledge all the study participants, services an help of the department of Laboratory Medicine, West China Hospital of Sichuan University.

\section{Funding:}

This article was funded by National Key R\&D Program or China (2020YFC2005600 and 2020YFC2005603).

Authors information

Affiliation

Department of Laboratory Medicine, West China Hospital of Sichuan University, 37 Guoxue Xiang, Chengdu, 610041, Sichuan, China.

Qianhui, Liu. Xin, Nie. Yong, He. Guixing, Li

Contributions 
Guixing, Li: Conceptualization, Methodology, Writing - Review \& Editing, Supervision, Project administration. Qianhui, Liu: Software, Formal analysis, Investigation, Data Curation, Writing - Original Draft, Visualization. Xin, Nie: Data Curation, Writing - Original Draft, Visualization. Yong, He: Writing Original Draft, Visualization.

\section{Ethics declarations}

Ethics approval and consent to participate

This retrospective study was approved by the Ethics Committee of West China Hospital of Sichuan University and all methods were performed in accordance with the relevant guidelines and regulations Consent for publication

Not applicable.

Competing Interests

The authors declare that they have no known competing financial interests or personal relationships that could have appeared to influence the work reported in this paper.

\section{References}

1. Wells, S.A., Asa, S.L., Dralle, H., Elisei, R., Evans, D.B., Gagel, R.F., Lee, N., Machens, A., Moley, J.F., Pacini, F., Raue, F., Frank-Raue, K., Robinson, B., Rosenthal, M.S., Santoro, M., Schlumberger, M., Shah, M., Waguespack, S.G.: Revised American Thyroid Association Guidelines for the Management of Medullary Thyroid Carcinoma. Thyroid 25(6), 567-610 (2015). doi:10.1089/thy.2014.0335

2. Lombardo, F., Baudin, E., Chiefari, E., Arturi, F., Bardet, S., Caillou, B., Conte, C., Dallapiccola, B., Giuffrida, D., Bidart, J.M., Schlumberger, M., Filetti, S.: Familial medullary thyroid carcinoma: Clinical variability and low aggressiveness associated with RET mutation at codon 804. Journal of Clinical Endocrinology \& Metabolism 87(4), 1674-1680 (2002). doi:10.1210/jcem.87.4.8403

3. Pacini, F., Castagna, M.G., Cipri, C., Schlumberger, M.: Medullary Thyroid Carcinoma. Clin. Oncol. 22(6), 475-485 (2010). doi:10.1016/j.clon.2010.05.002

4. Kebebew, E., Ituarte, P.H.G., Siperstein, A.E., Duh, Q.Y., Clark, O.H.: Medullary thyroid carcinoma Clinical characteristics, treatment, prognostic factors, and a comparison of staging systems. Cancer 88(5), 1139-1148 (2000). doi:10.1002/(sici)1097-0142(20000301)88:5<1139::Aid-cncr26>3.0.Co;2-z

5. Kuo, E.J., Sho, S.N., Li, N., Zanocco, K.A., Yeh, M.W., Livhits, M.J.: Risk Factors Associated With Reoperation and Disease-Specific Mortality in Patients With Medullary Thyroid Carcinoma. Jama Surg 153(1), 52-59 (2018). doi:10.1001/jamasurg.2017.3555

6. Ceolin, L., Duval, M.A.D., Benini, A.F., Ferreira, C.V., Maia, A.L.: Medullary thyroid carcinoma beyond surgery: advances, challenges, and perspectives. Endocrine-Related Cancer 26(9), R499-R518 (2019). doi:10.1530/erc-18-0574 
7. Trimboli, P., Treglia, G., Guidobaldi, L., Romanelli, F., Nigri, G., Valabrega, S., Sadeghi, R., Crescenzi, A., Faquin, W.C., Bongiovanni, M., Giovanella, L.: Detection rate of FNA cytology in medullary thyroid carcinoma: a meta-analysis. Clin. Endocrinol. 82(2), 280-285 (2015). doi:10.1111/cen.12563

8. Frank-Raue, K., Machens, A., Leidig-Bruckner, G., Rondot, S., Haag, C., Schulze, E., Lorenz, A., Kreissl, M.C., Dralle, H., Raue, F., Schmid, K.W.: Prevalence and Clinical Spectrum of Nonsecretory Medullary Thyroid Carcinoma in a Series of 839 Patients with Sporadic Medullary Thyroid Carcinoma. Thyroid 23(3), 294-300 (2013). doi:10.1089/thy.2012.0236

9. Pina, G., Dubois, S., Murat, A., Berger, N., Niccoli, P., Peix, J.L., Cohen, R., Guillausseau, C., Charrie, A., Chabre, O., Cornu, C., Borson-Chazot, F., Rohmer, V., Grp Tumeurs, E.: Is basal ultrasensitive measurement of calcitonin capable of substituting for the pentagastrin-stimulation test? Clin. Endocrinol. 78(3), 358-364 (2013). doi:10.1111/cen.12001

10. Niederle, M.B., Scheuba, C., Riss, P., Selberherr, A., Koperek, O., Niederle, B.: Early Diagnosis of Medullary Thyroid Cancer: Are Calcitonin Stimulation Tests Still Indicated in the Era of Highly Sensitive Calcitonin Immunoassays? Thyroid 30(7), 974-984 (2020). doi:10.1089/thy.2019.0785

11. Herrmann, B.L., Schmid, K.W., Goerges, R., Kemen, M., Mann, K.: Calcitonin screening and pentagastrin testing: predictive value for the diagnosis of medullary carcinoma in nodular thyroid disease. Eur. J. Endocrinol. 162(6), 1141-1145 (2010). doi:10.1530/eje-10-0111

12. Elisei, R., Bottici, V., Luchetti, F., Di Coscio, G., Romei, C., Grasso, L., Miccoli, P., lacconi, P., Basolo, F., Pinchera, A., Pacini, F.: Impact of routine measurement of serum calcitonin on the diagnosis and outcome of medullary thyroid cancer: Experience in 10,864 patients with nodular thyroid disorders. Journal of Clinical Endocrinology \& Metabolism 89(1), 163-168 (2004). doi:10.1210/jc.2003-030550

13. Pacini, F., Fontanelli, M., Fugazzola, L., Elisei, R., Romei, C., Dicoscio, G., Miccoli, P., Pinchera, A.: ROUTINE MEASUREMENT OF SERUM CALCITONIN IN NODULAR THYROID-DISEASES ALLOWS THE PREOPERATIVE DIAGNOSIS OF UNSUSPECTED SPORADIC MEDULLARY-THYROID CARCINOMA. Journal of Clinical Endocrinology \& Metabolism 78(4), 826-829 (1994). doi:10.1210/jc.78.4.826

14. Verbeek, H.H.G., de Groot, J.W.B., Sluiter, W.J., Kobold, A.C.M., van den Heuvel, E.R., Plukker, J.T.M., Links, T.P.: Calcitonin testing for detection of medullary thyroid cancer in people with thyroid nodules. Cochrane Database of Systematic Reviews(3) (2020). doi:10.1002/14651858.CD010159.pub2

15. Giannetta, E., Guarnotta, V., Altieri, B., Sciammarella, C., Guadagno, E., Malandrino, P., Puliani, G., Feola, T., Isidori, A.M., Colao, A.A.L., Faggiano, A., Nike: ENDOCRINE TUMOURS Calcitonin in thyroid and extra-thyroid neuroendocrine neoplasms: the two-faced Janus. Eur. J. Endocrinol. 183(6), R197R215 (2020). doi:10.1530/eje-20-0506

16. d'Herbomez, M., Caron, P., Bauters, C., Do Cao, C., Schlienger, J.L., Sapin, R., Baldet, L., Carnaille, B., Wemeau, J.L., French Group, G.T.E.: Reference range of serum calcitonin levels in humans: influence of calcitonin assays, sex, age, and cigarette smoking. Eur J Endocrinol 157(6), 749-755 (2007). doi:10.1530/EJE-07-0566

17. Bae, Y.J., Schaab, M., Kratzsch, J.: Calcitonin as Biomarker for the Medullary Thyroid Carcinoma. Recent results in cancer research. Fortschritte der Krebsforschung. Progres dans les recherches sur le 
cancer 204, 117-137 (2015). doi:10.1007/978-3-319-22542-5_5

18. Elisei, R.: Routine serum calcitonin measurement in the evaluation of thyroid nodules. Best Practice \& Research Clinical Endocrinology \& Metabolism 22(6), 941-953 (2008).

doi:10.1016/j.beem.2008.09.008

19. Toledo, S.P., Lourenco, D.M., Jr., Santos, M.A., Tavares, M.R., Toledo, R.A., Correia-Deur, J.E.: Hypercalcitoninemia is not pathognomonic of medullary thyroid carcinoma. Clinics (Sao Paulo) 64(7), 699-706 (2009). doi:10.1590/S1807-59322009000700015

20. Unluhizarci, K., Akgun, H., Oz, B., Karaca, Z., Tanriverdi, F., Kelestimur, F.: Patients with papillary thyroid carcinoma associated with high stimulated serum calcitonin levels. Endocrinol. Diabetes Metab. Case Rep., 4 (2017). doi:10.1530/edm-17-0085

21. Thiem, U., Marculescu, R., Cejka, D., Gessl, A., Borchhardt, K.: Low-dose calcium versus pentagastrin for stimulation of calcitonin in chronic hemodialysis patients: a pilot study. J Clin Endocrinol Metab 99(12), 4704-4711 (2014). doi:10.1210/jc.2014-1869

22. Becker, K.L., Nylen, E.S., White, J.C., Muller, B., Snider, R.H.: Clinical review 167 - Procalcitonin and the calcitonin gene family of peptides in inflammation, infection, and sepsis: A journey from calcitonin back to its precursors. Journal of Clinical Endocrinology \& Metabolism 89(4), 1512-1525 (2004). doi:10.1210/jc.2002-021444

23. Nozieres, C., Chardon, L., Goichot, B., Borson-Chazot, F., Hervieu, V., Chikh, K., Lombard-Bohas, C., Walter, T.: Neuroendocrine tumors producing calcitonin: characteristics, prognosis and potential interest of calcitonin monitoring during follow-up. Eur. J. Endocrinol. 174(3), 335-341 (2016). doi:10.1530/eje-15-0917

24. Mian, C., Perrino, M., Colombo, C., Cavedon, E., Pennelli, G., Ferrero, S., De Leo, S., Sarais, C., Cacciatore, C., Manfredi, G.I., Verga, U., lacobone, M., De Pasquale, L., Pelizzo, M.R., Vicentini, L., Persani, L., Fugazzola, L.: Refining Calcium Test for the Diagnosis of Medullary Thyroid Cancer: Cutoffs, Procedures, and Safety. Journal of Clinical Endocrinology \& Metabolism 99(5), 1656-1664 (2014). doi:10.1210/jc.2013-4088

25. Allelein, S., Ehlers, M., Morneau, C., Schwartz, K., Goretzki, P.E., Seppel, T., Feldkamp, J., Krieg, A., Knoefel, W.T., Kuebart, A., Haase, M., Dringenberg, T., Schmid, C., Schott, M.: Measurement of Basal Serum Calcitonin for the Diagnosis of Medullary Thyroid Cancer. Horm Metab Res 50(1), 23-28 (2018). doi:10.1055/s-0043-122237

26. Colombo, C., Verga, U., Mian, C., Ferrero, S., Perrino, M., Vicentini, L., Dazzi, D., Opocher, G., Pelizzo, M.R., Beck-Peccoz, P., Fugazzola, L.: Comparison of Calcium and Pentagastrin Tests for the Diagnosis and Follow-Up of Medullary Thyroid Cancer. Journal of Clinical Endocrinology \& Metabolism 97(3), 905-913 (2012). doi:10.1210/jc.2011-2033

27. Fugazzola, L., Di Stefano, M., Censi, S., Repaci, A., Colombo, C., Grimaldi, F., Magri, F., Pagotto, U., lacobone, M., Persani, L., Mian, C.: Basal and stimulated calcitonin for the diagnosis of medullary thyroid cancer: updated thresholds and safety assessment. J. Endocrinol. Invest., 11 (2020). doi:10.1007/s40618-020-01356-9 
28. Park, H., Park, J., Choi, M.S., Kim, J., Kim, H., Shin, J.H., Kim, J.H., Kim, J.S., Kim, S.W., Chung, J.H., Kim, T.H.: Preoperative Serum Calcitonin and Its Correlation with Extent of Lymph Node Metastasis in Medullary Thyroid Carcinoma. Cancers (Basel) 12(10) (2020). doi:10.3390/cancers12102894

29. Wolinski, K., Kaznowski, J., Klimowicz, A., Maciejewski, A., Lapinska-Cwojdzinska, D., Gurgul, E., Car, A.D., Fichna, M., Gut, P., Gryczynska, M., Ruchala, M.: Diagnostic value of selected biochemical markers in the detection of recurrence of medullary thyroid cancer - comparison of calcitonin, procalcitonin, chromogranin A, and carcinoembryonic antigen. Endokrynol Pol 68(4), 434-437 (2017). doi:10.5603/EP.a2017.0038

30. Chen, L., Zhao, K., Li, F.X., He, X.H.: Medullary Thyroid Carcinoma With Elevated Serum CEA and Normal Serum Calcitonin After Surgery: A Case Report and Literature Review. Frontiers in Oncology 10 (2020). doi:10.3389/fonc. 2020.526716

31. Meijer, J.A.A., le Cessie, S., van den Hout, W.B., Kievit, J., Schoones, J.W., Romijn, J.A., Smit, J.W.A.: Calcitonin and carcinoembryonic antigen doubling times as prognostic factors in medullary thyroid carcinoma: a structured meta-analysis. Clin. Endocrinol. 72(4), 534-542 (2010). doi:10.1111/j.13652265.2009.03666.x

\section{Figures}




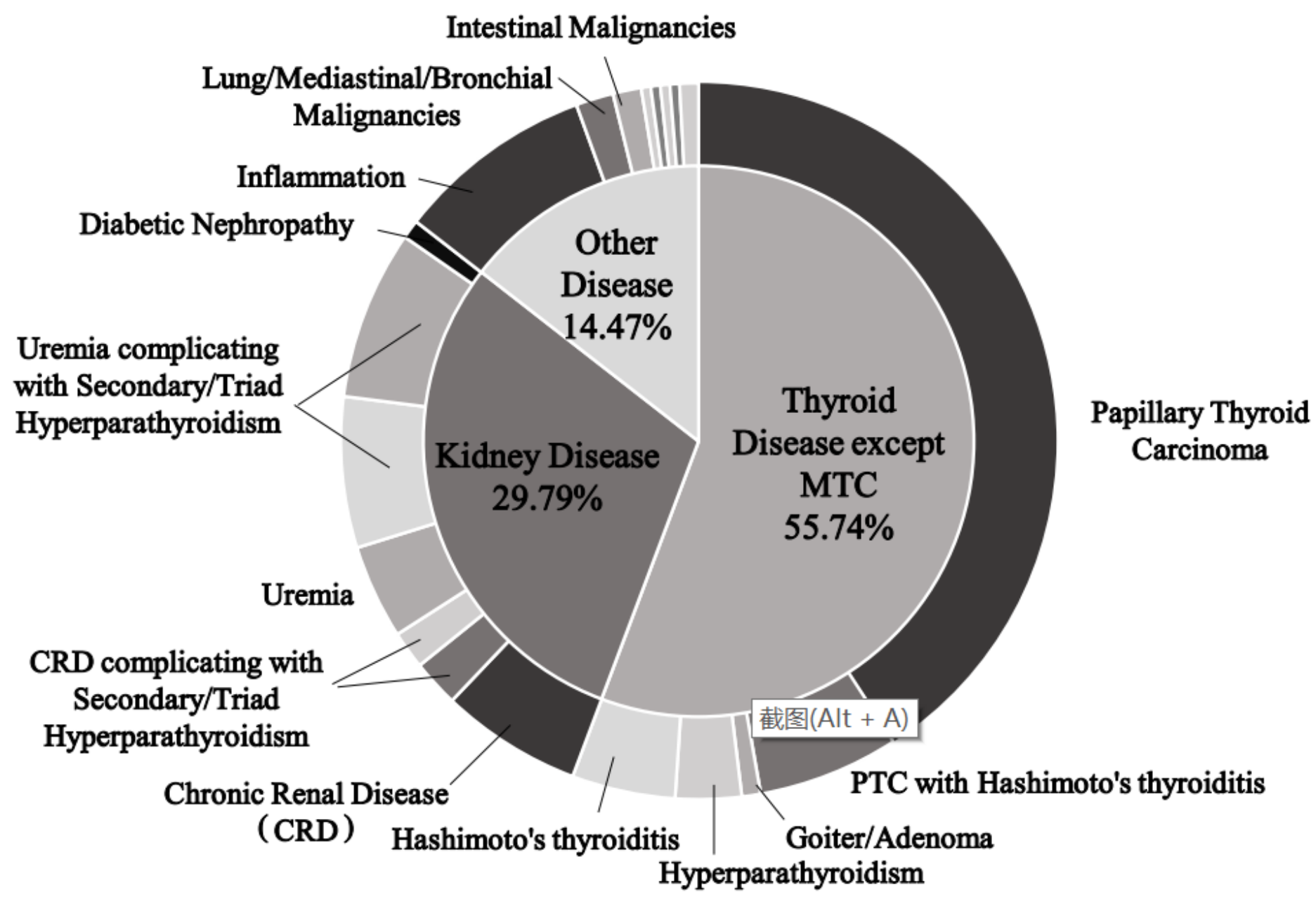

Figure 1

Disease distribution of the 235 non-MTC patients with elevated serum calcitonin 

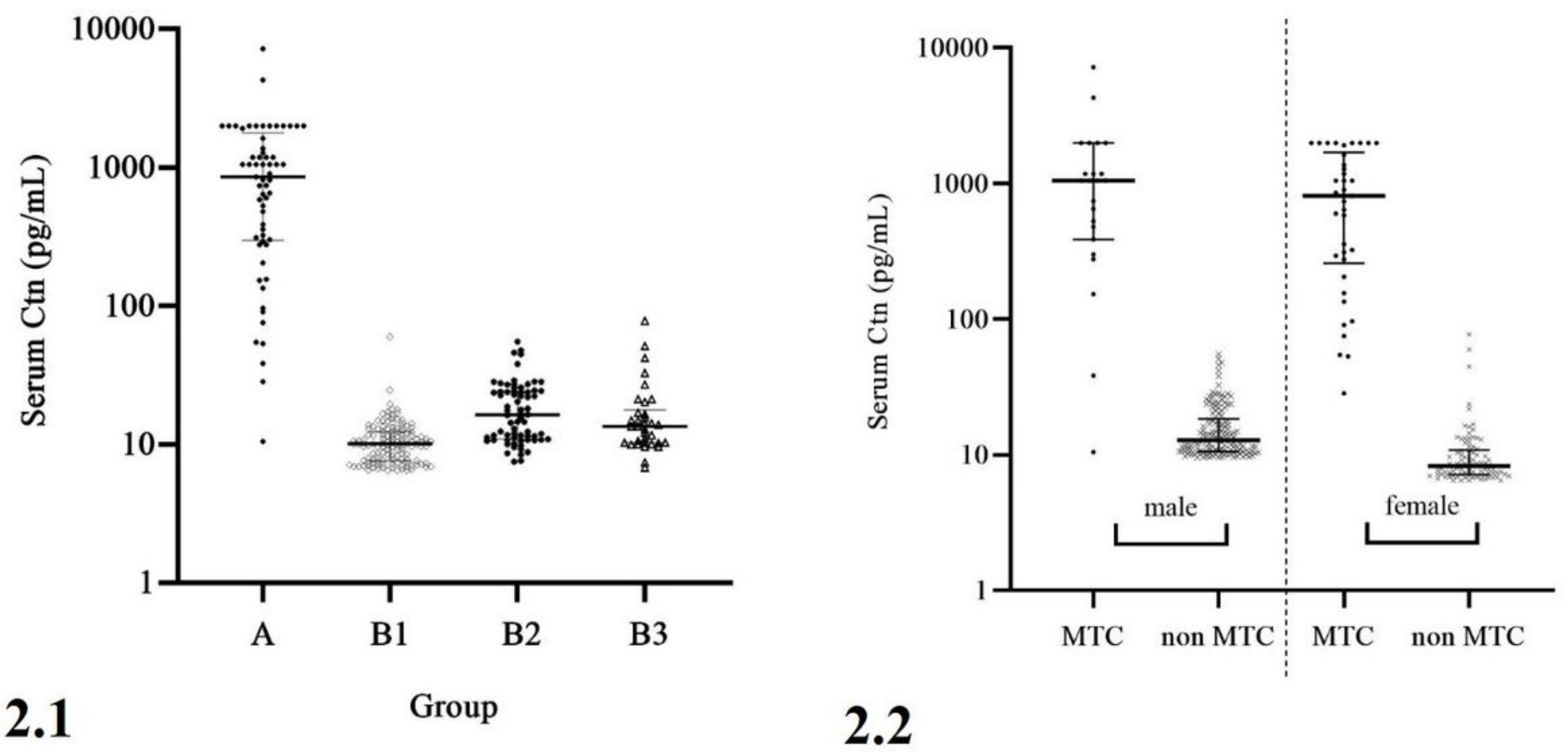

2.2

Figure 2

Figure 2.1 Distribution of serum calcitonin levels among the four groups. Figure 2.2 Distribution of serum calcitonin in the MTC and non-MTC groups based on sex. 
a

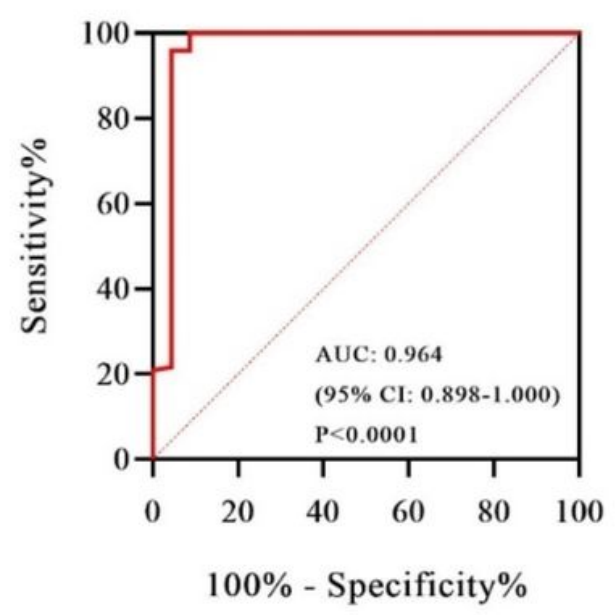

c Female

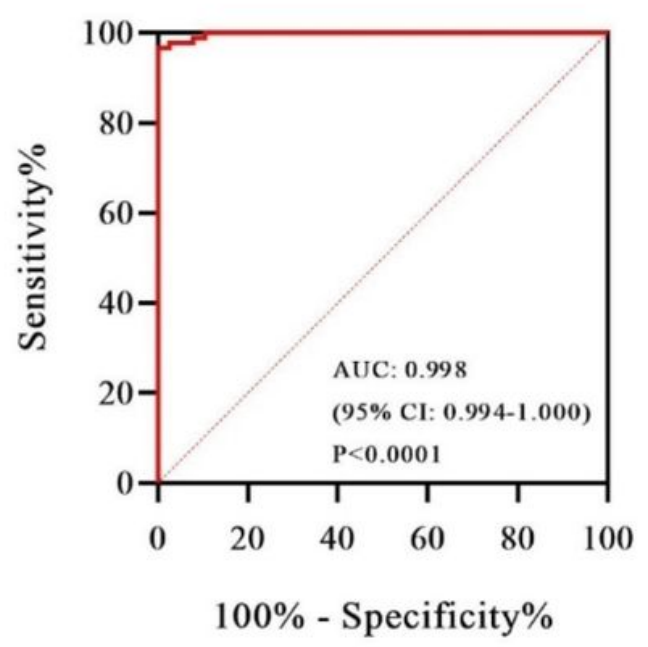

b

Male

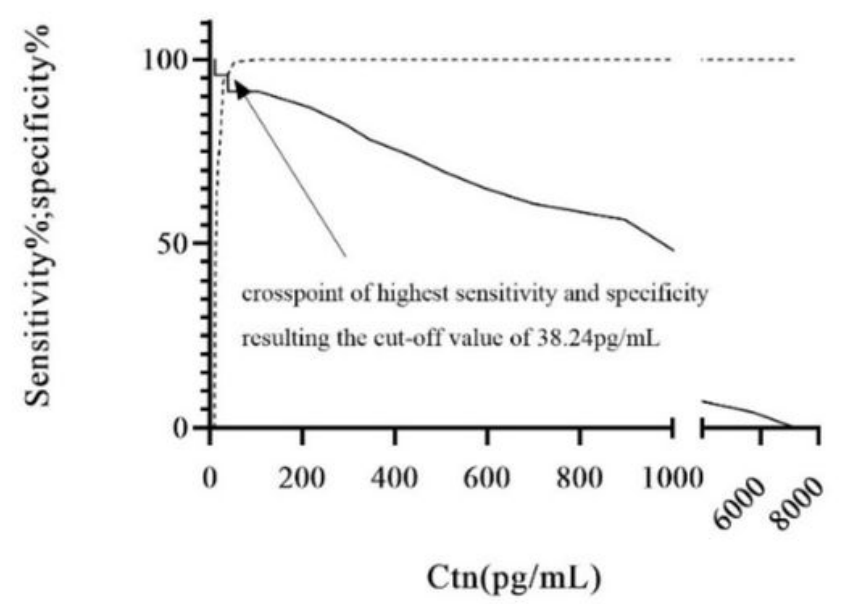

Female

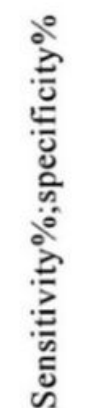

d

- sensitivity\%

..... specificity\%

Figure 3

ROC curves of calcitonin for males and females 


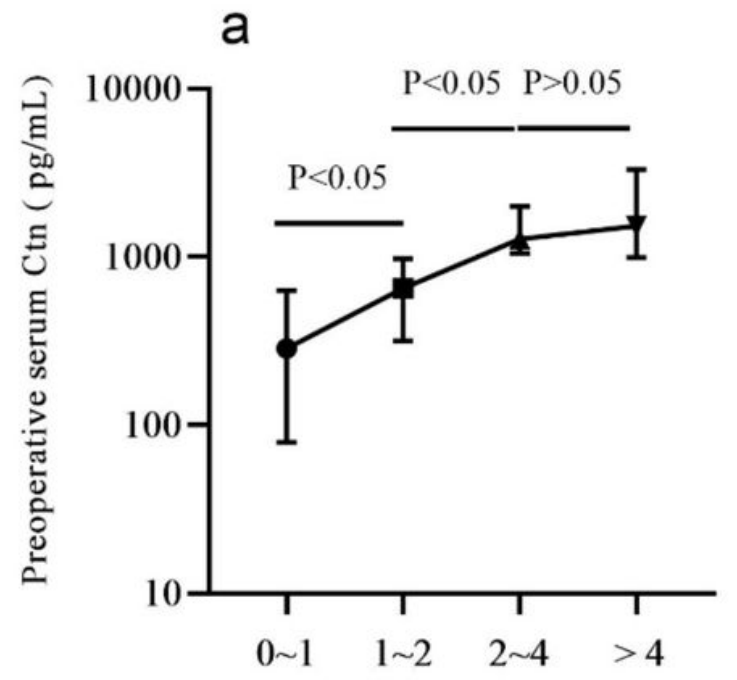

The largest tumor diameter $(\mathrm{cm})$

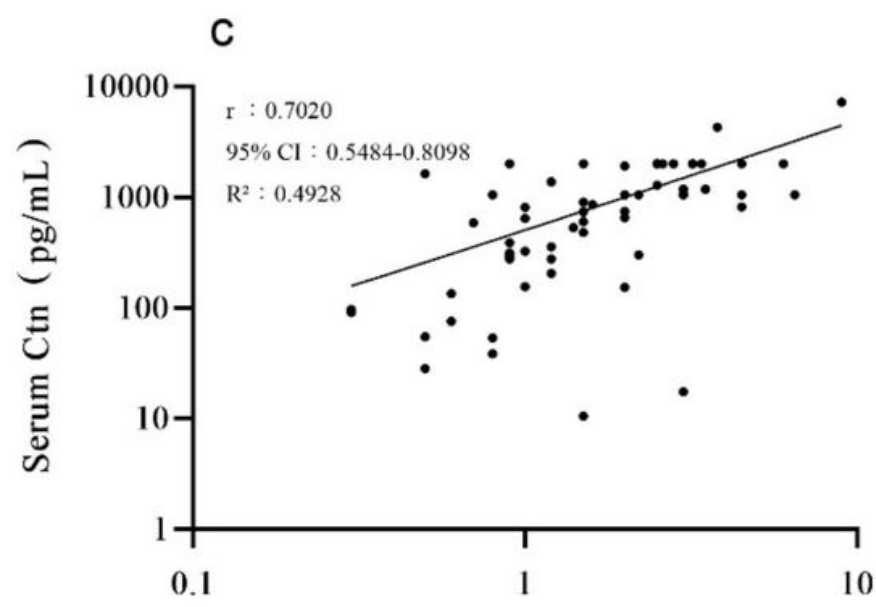

The largest tumor diameter $(\mathrm{cm})$

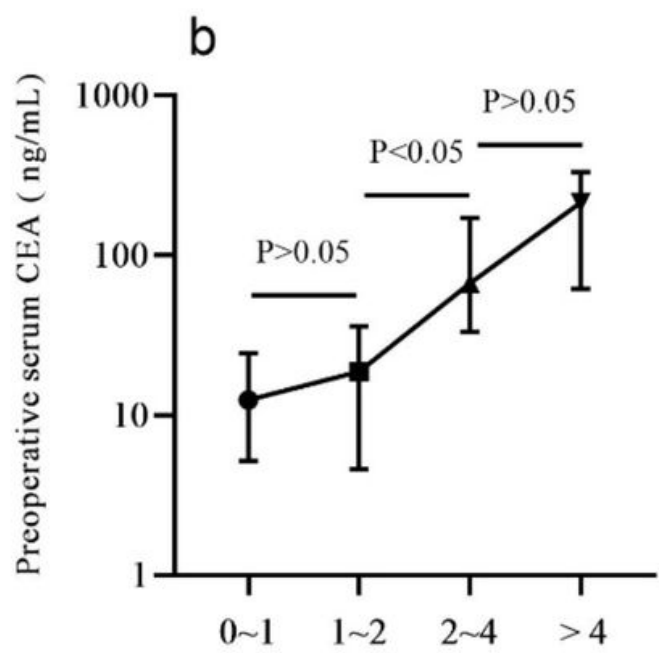

The largest tumor diameter(cm)

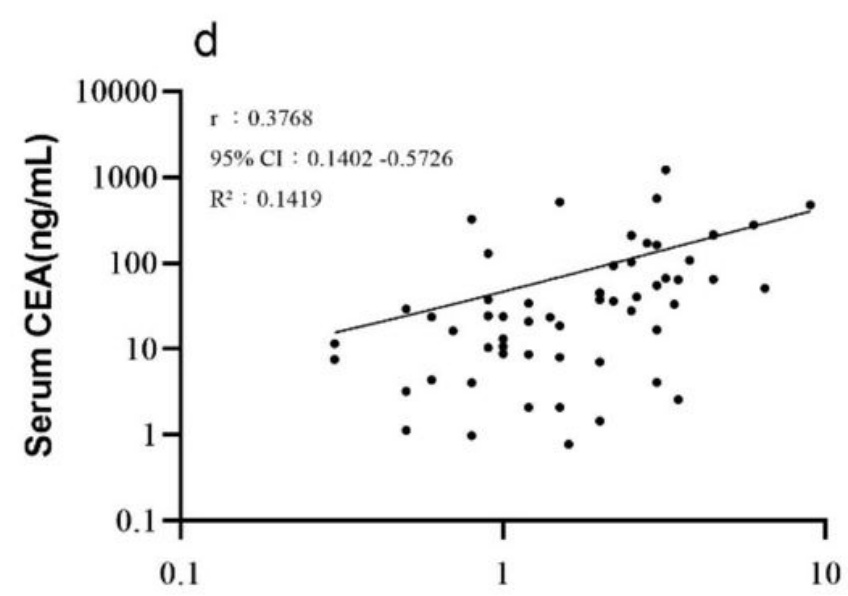

The largest tumor diameter(cm)

Figure 4

$a$ and $b$ : The comparison of serum calcitonin and carcinoembryonic antigen values according to tumor size. c and d: The relationship of serum calcitonin, carcinoembryonic antigen and tumor size. 
a

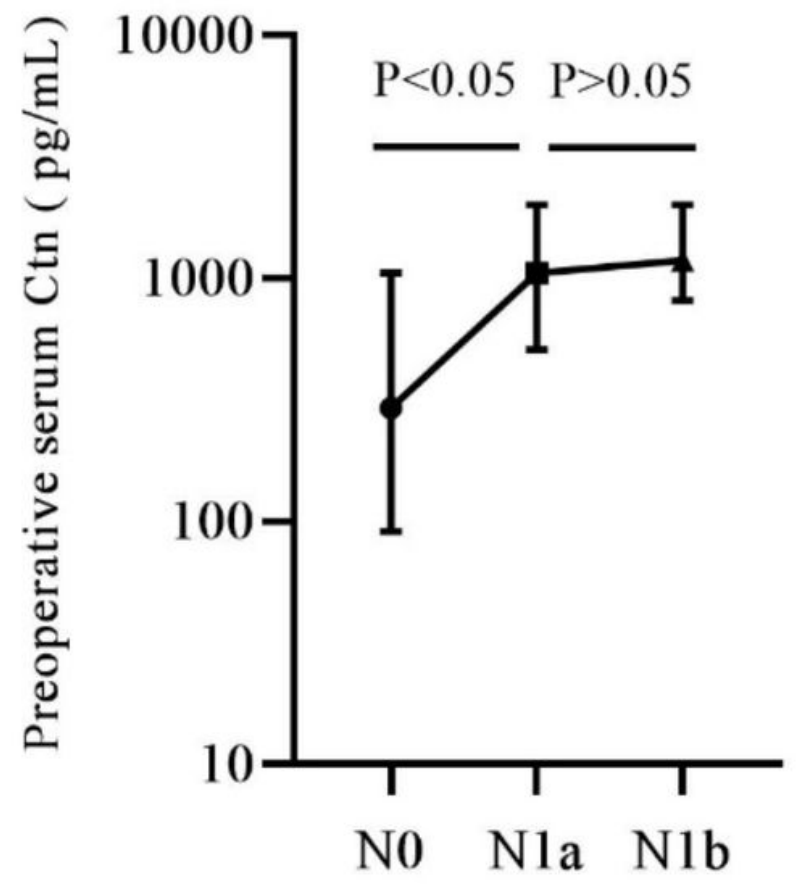

Lymph node staging

b

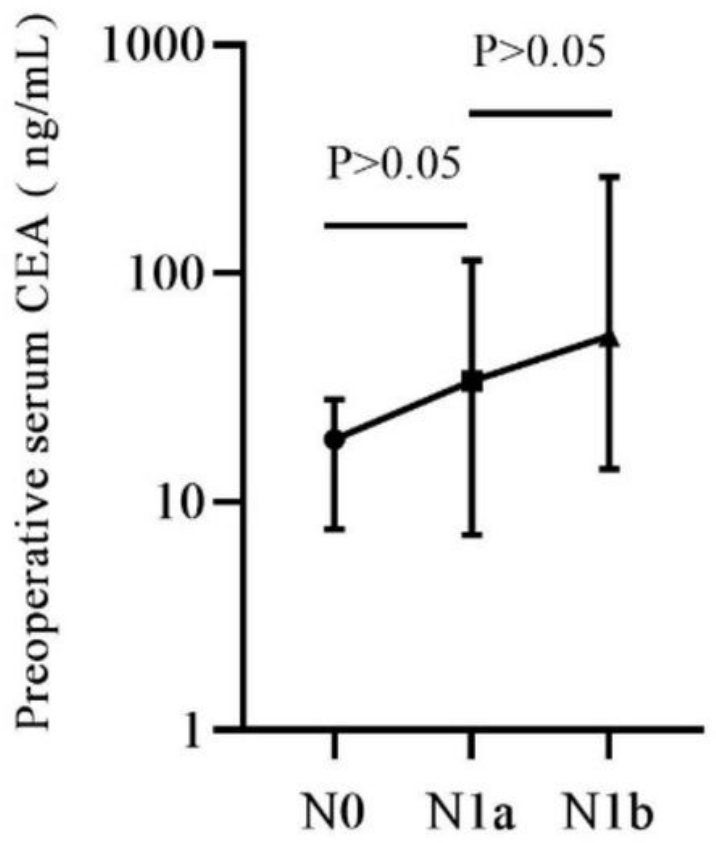

Lymph node staging

Figure 5

Comparison of serum calcitonin and carcinoembryonic antigen values according to lymph node stage. 